\title{
Lyapunov stability of singular planar systems related to dispersion-managed solitons in optical fiber
}

\author{
Zaitao Liang ${ }^{1}$, Xin $\mathrm{Li}^{1}$, Shengjun $\mathrm{Li}^{2}$, Xuemeng Shan ${ }^{1}$, and Feng Wang ${ }^{3}$ \\ ${ }^{1}$ Anhui University of Science and Technology \\ ${ }^{2}$ Hainan University \\ ${ }^{3}$ Changzhou University
}

April 11, 2021

\begin{abstract}
In this paper, we consider two singular planar differential systems which can describe the evolution of the optical pulse width and chirp for the so-called dispersion-managed solitons. Based on the method of third order approximation in combination with some quantitative information obtained by the upper-lower solutions method and the averaging method, some results on the existence and Lyapunov stability of the periodic solutions are obtained. Moreover, the formula of the first twist coefficient and a stability criterion of a nonlinear differential equation are also established.
\end{abstract}

\section{Hosted file}

LLLSW.pdf available at https://authorea.com/users/407163/articles/517559-lyapunov-stabilityof-singular-planar-systems-related-to-dispersion-managed-solitons-in-optical-fiber 